\title{
Probing high-mass star formation in the outflow source G12.42+0.50
}

\author{
Anandmayee Tej \\ Indian Institute of Space Science and Technology, Thiruvananthapuram 695 547, Kerala, India
}

\begin{abstract}
This talk is based on the paper "Initial phases of high-mass star formation: A multiwavelength study towards the extended green object G12.42+0.50" by Namitha Issac, Anandmayee Tej, Tie Liu, Watson Varricatt, Sarita Vig, Ishwara Chandra $\mathrm{CH}$ and Mathias Schultheis and has been accepted for publication in MNRAS. A multiwavelength study of the extended green object G12.42+0.50 is presented. The associated ionized, dust, and molecular components of this source is probed using various observations at near- and farinfrared, submillimeter and radio wavelengths. The radio continuum emission mapped at 1390 and $610 \mathrm{MHz}$ calls for a scenario of co-existence of an ultracompact (UC) $\mathrm{H}$ II region with an ionized jet possibly powered by the same young stellar object (YSO). Shock-excited lines of $\mathrm{H}_{2}$ and [Fe II] seen in the NIR spectra provide support to this picture. Study of the gas kinematics of the molecular cloud reveals the presence of both infall activity and large-scale molecular outflow, suggesting an early stage of massive star formation in G12.42+0.50.
\end{abstract}

Keywords: HII regions - jets and outflows - individual objects (G12.42+0.50)

\section{Introduction}

Massive stars play a crucial role in the evolution of the galaxies and hence the universe through their radiative, mechanical and chemical feedback. The strong winds, powerful radiations and supernova events are a source of mixing and turbulence in the ISM of galaxies. Inspite of this, most of the processes involved in their formation are less understood in comparison to their low-mass counterparts. Various competing theories have been proposed to explain the formation mechanism of high-mass stars, such as Core Accretion, Competitive Accretion and Protostellar Merger. The dearth of observations due to rarity of sources, large distances, influenced environment and high extinction hinder the development of a proper observational database to validate the proposed theory and propose the preferred mode of formation, if any. The challenge lies in probing the very early stages of high-mass star formation

The large-scale Spitzer Galactic Legacy Infrared Mid-Plane Survey Extraordinaire (GLIMPSE) (Benjamin et al. 2003) provides an excellent database of objects showing enhanced and extended emission in the IRAC $4.5 \mu \mathrm{m}$ band. Following the conventional color-coding of the GLIMPSE colour-composite images, this class of sources was labelled 'green fuzzies' or 'extended green objects' (EGOs) by Chambers et al. (2009) and Cyganowski et al. (2008), respectively. Several studies have aimed at ascertaining the nature of these objects. Studies over the last decade converge towards 
a picture of EGOs being associated with protostellar outflows/jets from massive young stellar objects (MYSO), thus offering an unique sample of sources to probe the early phases of massive star formation.

In our study, we target the EGO G12.42+0.50 catalogued as a "possible" outflow candidate and associated with the infrared source IRAS 18079-1756. G12.42+0.50 is located at a kinematic distance of $2.4 \mathrm{kpc}$ (Chen et al. 2010). It is designated as an ultracompact (UC) H II region (Jaffe et al. 1984; Wu et al. 2007) and has been observed as a part of several surveys such as the Millimeter Astronomy Legacy Team $90 \mathrm{GHz}$ (MALT90; Foster et al. 2011; Jackson et al. 2013) and the $6 \mathrm{~cm}$ Red MSX Source survey (Urquhart et al. 2009). Here, we scrutinize G12.42+0.50 adopting a multiwavelength approach using observations and archival data sets from radio wavelengths to the NIR bands.

\section{Emission from ionized gas}

To probe the nature of the ionized gas associated with G12.42+0.50, we carried out low-frequency radio observations at 1390 and $610 \mathrm{MHz}$ using the Giant Meterwave Radio Telescope (GMRT) located at Pune, India. Data reduction is performed using the NRAO Astronomical Image Processing Software (AIPS) following standard procedures.

The radio continuum maps at 1390 and $610 \mathrm{MHz}$ are shown in Fig. 1. The $1390 \mathrm{Mhz}$ map reveals a linear structure in the north-east south-west direction with two distinct components, labeled R1 and R2, where R1 seems to be well resolved while, R2 seems to be barely resolved. Both these components are also visible in the high-resolution $6 \mathrm{~cm}(5 \mathrm{GHz})$ map (Urquhart et al. 2009). However the lower resolution $610 \mathrm{MHz}$ map shows a single, almost spherical morphology with the peak coinciding with the position of R1 and a discernible elongation towards R2.
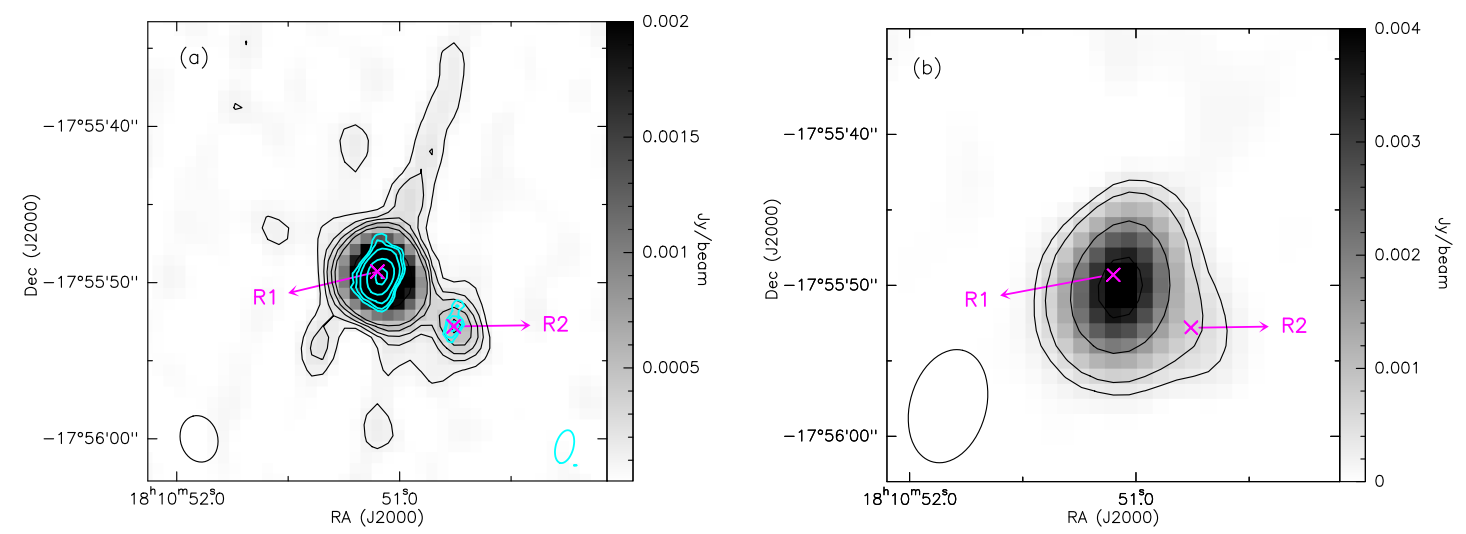

Figure 1: (a) High resolution radio continuum map of G12.42+0.50 at $1390 \mathrm{MHz}$ with the contour levels 3, 6, 9, 18, 63, 150 and 172 times $\sigma(\sigma \sim 29.7 \mu \mathrm{Jy} / \mathrm{beam})$. Positions of R1 and R2 are also labelled. The contours of the $6 \mathrm{~cm}$ radio map are overlaid in cyan with the contour levels 3, 4, 6, 12, 21 and $24 \sigma(\sigma \sim 0.15 \mathrm{mJy} / \mathrm{beam})$. (b) The radio continuum map $610 \mathrm{MHz}$ with contour levels $3,6,18,38$ and 60 times $\sigma(\sigma \sim 94 \mu \mathrm{Jy} / \mathrm{beam})$. The positions of the two radio peaks detected in the $1390 \mathrm{MHz}$ map is indicated by ' $\mathrm{x}$ '. The restoring beams in the 1390 and $610 \mathrm{MHz}$ bands are represented as open ellipses towards the bottom-left of each image and of the $6 \mathrm{~cm}$ map is represented as an open cyan ellipse towards the bottom-right in (a). This figure is taken from Issac et al. (2019) .

In order to get a better picture of the nature of the ionized emission, we generate the spectral index map using the 1390 and $610 \mathrm{MHz}$ maps. The spectral index, $\alpha$, is defined as $S_{\nu} \propto \nu^{\alpha}$, where $S_{\nu}$ is the flux density at frequency $\nu$. The generated spectral index map and the corresponding error map is shown in Fig. 2. It can be seen that the spectral index varies between 0.3 and 0.9 indicating that 
the region is dominated by thermal bremsstrahlung emission of varying optical depth. Given these values of the spectral index and the morphology of the ionized emission, we explore two scenarios to explain the nature of the radio emission.
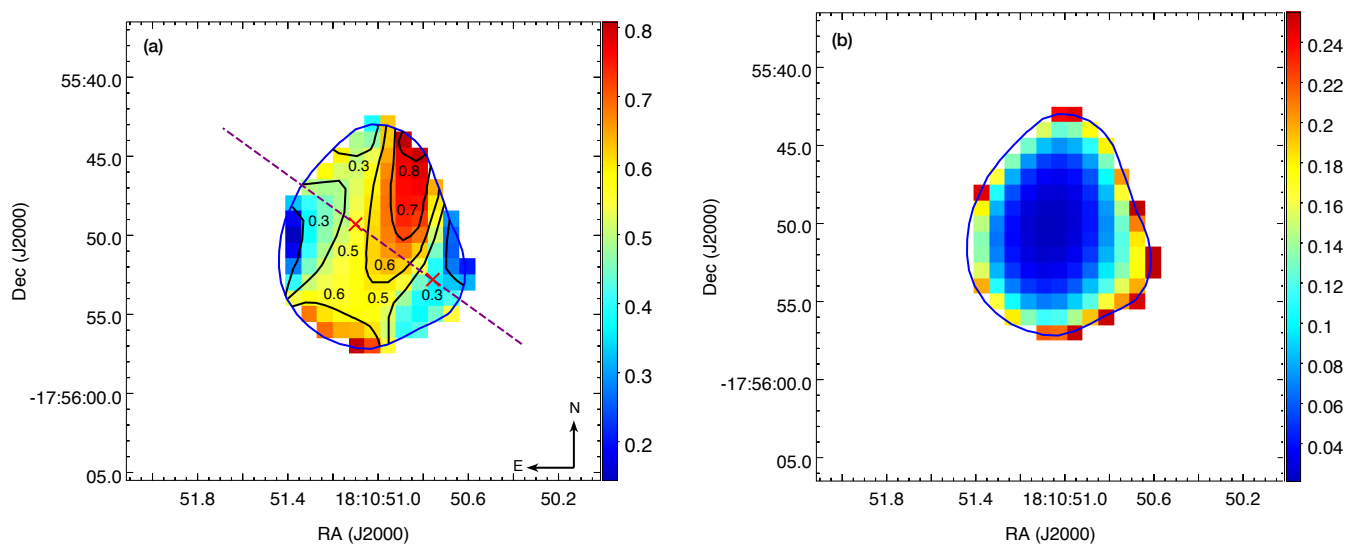

Figure 2: (a) Spectral index map of G12.42+0.50 between 1390 and $610 \mathrm{MHz}$. Black curves represent the spectral index levels. The blue contour shows the $5 \sigma\left(\sigma \sim 0.4 \times 10^{-4} \mathrm{Jy} / \mathrm{beam}\right)$ level of the $610 \mathrm{MHz}$ map used to construct the spectral index map. The red 'x's mark the positions of the radio components, R1 and R2. The dashed purple line indicates the possible direction of the ionized jet. The spectral index varies from 0.3 to 0.7 along the possible jet axis. The error map is shown in (b). This figure is taken from Issac et al. (2019).

\subsection{UC H II region}

Given the compact and spherical morphology of the radio component, R1, we first investigate under the UC H II region framework. Under this picture, assuming the continuum emission at $1390 \mathrm{MHz}$ to be optically thin and arising from a isothermal, homogeneous medium, we derive the Lyman continuum photon flux $\left(N_{L y}\right)$ that translates to a spectral type of B1 - B0.5 for the ionizing star. The radius and dynamical age of the UC H II region associated with the component R1 is estimated to be $0.01 \mathrm{pc}$ and $0.4 \times 10^{3} \mathrm{yr}$, respectively, suggesting an UC $\mathrm{H}$ II region at a very early stage of evolution.

\subsection{Possible thermal jet}

Notwithstanding the compelling evidence supporting the UC H II region scenario of R1, we investigate an alternate scenario along the lines of a possible thermal jet, given the very nature of G12.42+0.50 identified as an EGO and hence likely to be associated with jets/outflows. Further, several observed features are consistent with the characteristics of jets discussed in Anglada (1996) and Rodriguez (1997). The ionized emission associated with G12.42+0.50 displays a linear morphology in the direction of the radio components $\mathrm{R} 1$ and $\mathrm{R} 2$ along which the spectral index varies in the range of $0.3-0.7$. Such values are consistent with the radio continuum emission due to thermal freefree emission from ionized jets. Also the presence of shock-excited $\mathrm{H}_{2}$ and [Fe II] lines at NIR and the association of G12.42+0.50 with large scale molecular outflow (Section 5) lend support to this scenario.

With strong evidences supporting both competing scenarios, we consider a picture of co-existence of the UC H II with the ionized jet. We find support for this picture from recent studies that speculate the co-existence scenario, where both the UC H II region and the ionized jet are powered by the same YSO (Beltrán et al. 2016; Guzmán et al. 2016). This suggests an interesting transitional phase where 
the UC H II has just begun to form while infall and outflow processes of the main accretion phase are still ongoing.

\section{Emission from shock indicators}

With growing evidence associating EGOs with MYSOs, several mechanisms like shock emission in outflows, fluorescent emission or scattered continuum from the MYSOs are explored to identify the spectral carriers at $4.5 \mu \mathrm{m}$ and NIR wavelengths. The shocked emission scenario suggests the spectral carriers to be molecular and atomic shock indicators like $\mathrm{H}_{2}$ and [Fe II] as well as broad CO bandheads. A few studies have focused at ascertaining the nature of emission at the NIR bands (Lee et al. 2012, 2013; Caratti o Garatti et al. 2015).

In order to carefully examine the NIR picture, we inspect the $\mathrm{H}_{2}$ line emission retrieved from the UWISH2 survey and the [Fe II] line emission from the observations made using the 3.8-m United Kingdom Infrared Telescope (UKIRT) Wide-Field Camera. The continuum subtracted $\mathrm{H}_{2}$ and [Fe II] images are depicted in Fig. 3. It can be seen that the $\mathrm{H}_{2}$ emission is extended towards the peak of the $4.5 \mu \mathrm{m}$ emission coincident with the radio component $\mathrm{R} 1$. The continuum subtracted [Fe II] image also shows a weak extended emission towards the brighter part of the $\mathrm{H}_{2}$ emission.
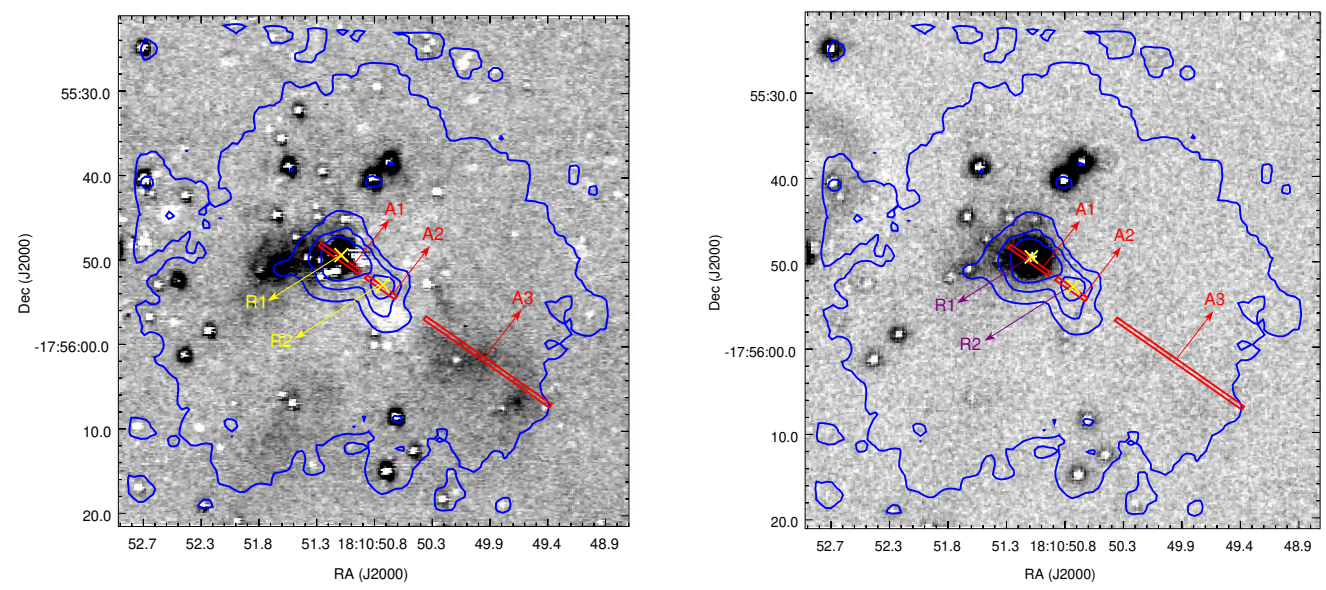

Figure 3: (a) Continuum subtracted $\mathrm{H}_{2}$ and (b) [Fe II] image towards G12.42+0.50. The positions of the identified radio components R1 and R2 are indicated. The blue contours represent the $4.5 \mu \mathrm{m}$ emission with the levels 3, 60, 120, and 220 $\sigma(\sigma \sim 1.5 \mathrm{MJy} / \mathrm{sr})$. The red rectangles show the orientation of the slit and denote the apertures used for spectra extraction. This figure is taken from Issac et al. (2019).

To understand further the results from the narrow-band imaging, we probe G12.42+0.50 with NIR spectroscopy using the UIST instrument on UKIRT. The spectra were obtained at two grism set-ups, namely $H K$ and $K L$. The data reduction is performed by the ORAC-DR pipeline at UKIRT, followed by standard procedures employed for spectral data reduction. The orientation of the slit is given in Fig. 3 ensuring that regions covering the radio components and the extended $\mathrm{H}_{2}$ line emission, along with the detached elongated nebulosity towards the south-west, are sampled. The $H K$ - and $K L$ spectra are extracted towards three apertures (marked in Fig. 3). The aperture A1 samples the radio component $\mathrm{R} 1$ and the extended $\mathrm{H}_{2}$ emission seen towards the north-east of the $4.5 \mu \mathrm{m}$ peak. The $H K$ and $K L$ spectra extracted are shown in Fig. 4. The spectra show clear detection of six emission lines of molecular $\mathrm{H}_{2}$ with the $(1-0) \mathrm{S}(1)$ line at $2.122 \mu \mathrm{m}$ being the most prominent feature. A weak [Fe II] line is also detected at $1.644 \mu \mathrm{m}$, towards the blue part of the $H K$ specturm. In addition to the 
emission lines, the slope of the continuum is seen to rise towards the red part of the $H K$ spectrum, indicating a highly embedded source. The $\mathrm{H}_{2}$ lines detected in $\mathrm{G} 12.42+0.50$ originate from the upper vibrational level, $v=1$, suggesting a low level of excitation. The absence of emission from higher vibrational levels supports the shock-excited origin of the detected $\mathrm{H}_{2}$ lines. No lines are detected above the noise level in the spectra extracted towards the radio component R2 (aperture A2) and the detached, extended nebulosity towards the south-west (aperture A3).
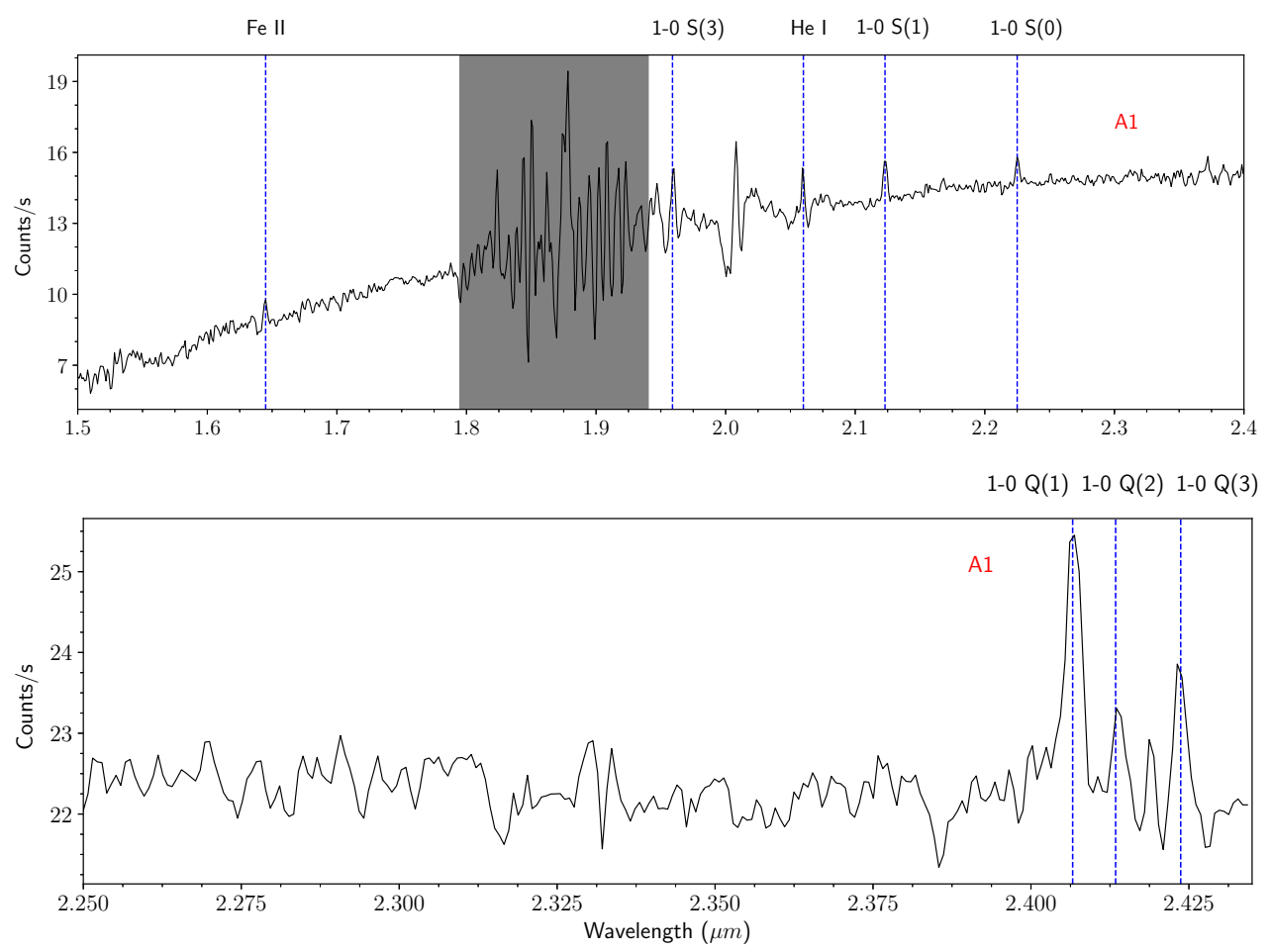

Figure 4: The $H K$ (upper panel) and $K L$ (lower panel) band spectrum of G12.42+0.50 extracted over the apertures A1. The shaded area marks the region of poor sky transparency. The identified spectral lines along aperture A1 are marked over the spectra. This figure is taken from Issac et al. (2019).

\section{Cold dust emission}

The nature of the cold dust associated with G12.42+0.50 is probed using the Herschel FIR bands covering the wavelength range $160-500 \mu \mathrm{m}$ and the combined ATLASGAL-Planck data at $870 \mu \mathrm{m}$. The dust temperature and the line-of-sight molecular hydrogen column density maps are generated by pixel-by-pixel modified blackbody model fitting of the functional form (Ward-Thompson \& Robson 1990; Battersby et al. 2011)

$$
S_{\nu}(\nu)-I_{b g}(\nu)=B_{\nu}\left(\nu, T_{d}\right) \Omega\left(1-e^{-\tau_{\nu}}\right)
$$

where

$$
\tau_{\nu}=\mu_{H_{2}} m_{H} \kappa_{\nu} N\left(H_{2}\right)
$$

Here $S_{\nu}$ is the observed flux density, $B_{\nu}\left(\nu, T_{d}\right)$ is the Planck function, $T_{d}$ is the dust temperature, $\Omega$ is the solid angle in steradians, from where the flux is measured (solid angle subtended by a $14^{\prime \prime} \times 14^{\prime \prime}$ pixel), $\mu_{H_{2}}$ is the mean molecular weight (taken as 2.8 ; Kauffmann et al. 2008), $\kappa_{\nu}$ is 
the dust opacity and $N\left(H_{2}\right)$ is the hydrogen column density. For opacity we assume the function $\kappa_{\nu}=0.1(\nu / 1000 \mathrm{GHz})^{\beta} \mathrm{cm}^{2} \mathrm{~g}^{-1}$, where $\beta$ is the dust emissivity spectral index which is fixed at 2.0. The spectral energy distribution modelling for each pixel is carried out keeping the dust temperature and hydrogen column density as free parameters. The generated hydrogen column density and temperature maps are shown in Fig. 5.

A dense and bright region is seen towards G12.42+0.50 and identified as the clump, $\mathrm{C} 1$ in the column density map. Apart from $\mathrm{C} 1$, other regions of density enhancements are also visible towards the west and south-west of G12.42+0.50, where additional 11 clumps were identified above a $3 \sigma$ threshold. The temperature map reveals two high temperature regions, one coinciding with $\mathrm{G} 12.42+0.50$, and the other towards the north-west of $\mathrm{C} 1$. The warmest temperature is found to be $28.8 \mathrm{~K}$ located within $\mathrm{C} 1$ and low dust temperatures are seen towards the other clumps. Both maps show filamentary structures towards the south-west which appear to converge at C1 suggesting a hub-filament morphology.
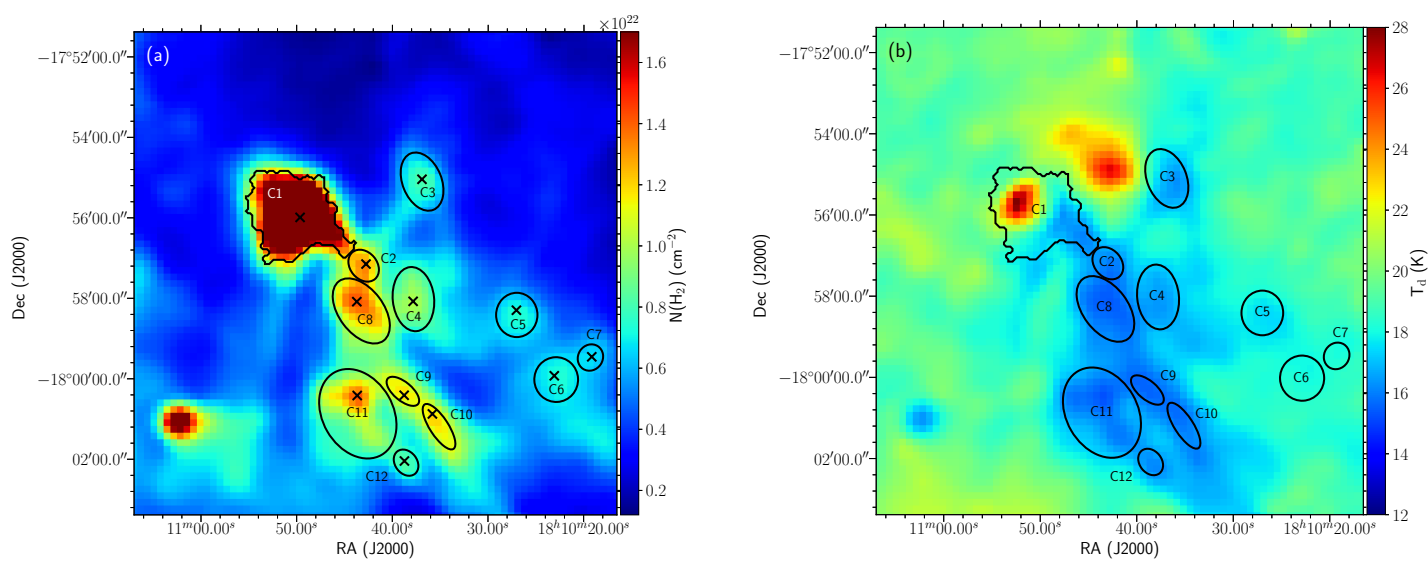

Figure 5: (a) Column density and (b) Temperature maps towards G12.42+0.50 generated using the Herschel FIR data and the ATLASGAL-Planck data. The Clumpfind retrieved clump $\mathrm{C} 1$ and the visually identified clumps from the column density map are marked on the maps. The ' $x$ 's mark the positions of the peak column densities of each clump in the column density map. This figure is taken from Issac et al. (2019).

From the SMA $1.1 \mathrm{~mm}$ observations, three cores are identified and shown in Figure 7. SMA1 and SMA2 show up as dense, compact cores possibly in a binary system. They are located towards the north-east edge of radio component, R1. SMA3, on the other hand, looks more like a clumpy region of density enhancement. SMA1 and SMA2 are found to be potential high-mass star-forming cores based on their mass and radius estimations.

\section{Kinematic signatures of gas motion}

The study of molecular line emission is vital in probing the kinematic and chemical structure of a molecular cloud as well as its evolutionary stage. In order to investigate theses aspects of the starforming region associated with G12.42+0.50, we make use of the data from the MALT90 survey and JCMT archives.

The $\mathrm{HCO}^{+}(1-0)$ molecular line displays double-peaked line profiles with a self-absorption dip at the LSR velocity that is derived from the optically thin $\mathrm{H}^{13} \mathrm{CO}^{+}$transition to be $18.3 \mathrm{~km} \mathrm{~s}^{-1}$. The blueshifted emission peak is stronger than the red-shifted peak. Such a blue-skewed profile is a characteristic signature of infall activity. To probe the gas motion in the entire clump associated 
with $\mathrm{G} 12.42+0.50$, we created a grid map of the $\mathrm{HCO}^{+}$line profile covering the whole clump. It is depicted in Fig. 6. It is evident that the $\mathrm{HCO}^{+}$spectrum displays blue-skewed line profiles in all the grids within the $870 \mu \mathrm{m}$ contour, indicating that the clump is in global collapse.

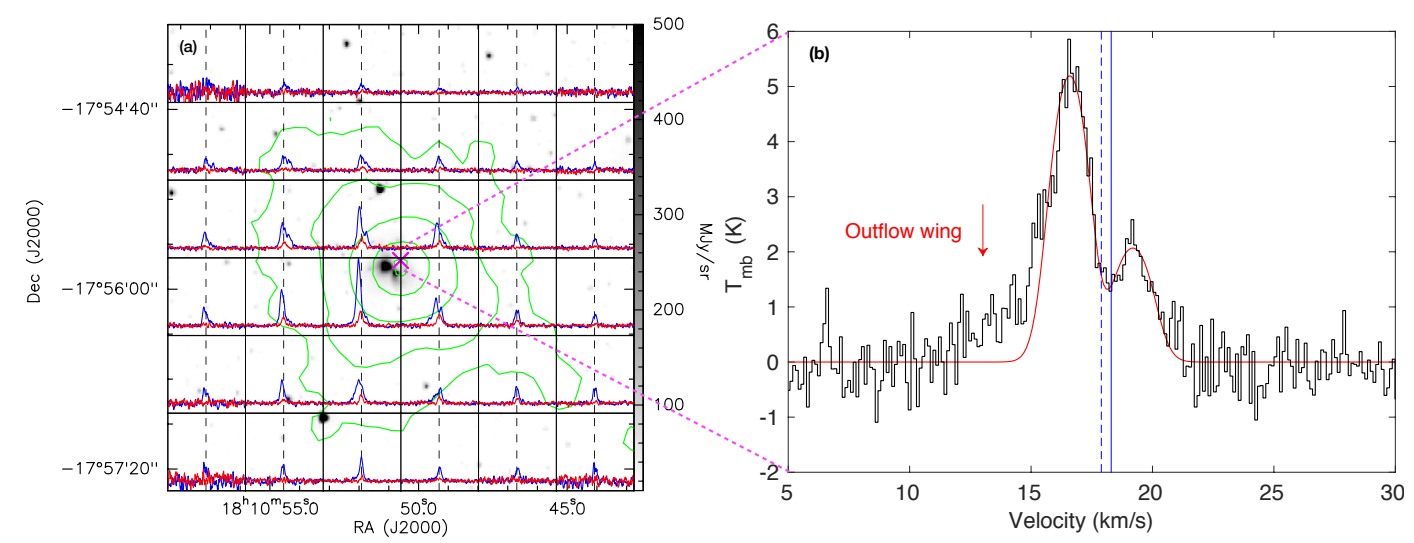

Figure 6: (a) The grey scale shows the $4.5 \mu \mathrm{m}$ map. The green contours represent the $870 \mu \mathrm{m}$ emission. The contour levels are 3, 9, 27, 72 and 108 times $\sigma(\sigma \sim 0.06 \mathrm{Jy} / \mathrm{beam})$. The $\mathrm{HCO}^{+}$ spectrum shown in blue and the $\mathrm{H}^{13} \mathrm{CO}^{+}$spectrum shown in red are overlaid. The dashed vertical lines indicate the LSR velocity estimated by averaging the peak positions of the $\mathrm{H}^{13} \mathrm{CO}^{+}$line in all the regions where the line is detected. The peak position of the $870 \mu \mathrm{m}$ emission is marked by a magenta ' $\mathrm{x}$ '. (b) The $\mathrm{HCO}^{+}$spectrum extracted towards the $870 \mu \mathrm{m}$ peak. The solid blue line represents the LSR velocity, $18.3 \mathrm{~km} \mathrm{~s}^{-1}$, derived from the optically-thin $\mathrm{H}^{13} \mathrm{CO}^{+}$line. The red arrow points to a blue wing which could indicate a possible molecular outflow. This figure is taken from Issac et al. (2019).

Massive molecular outflows, most often found in star-forming regions, are generally found to be co-existent with ionized jets. The outflows are believed to be driven by the jets that entrain the gas and dust from the surrounding molecular cloud. Broad wings of $\mathrm{HCO}^{+}$lines are generally accepted signatures of outflow as is seen for the $\mathrm{HCO}^{+}$line in $\mathrm{G} 12.42+0.50$. The rotational transition lines of $\mathrm{CO}$ are also well-known tracers of outflow activity. We probe the outflow kinematics of the molecular cloud associated with G12.42+0.50 using the $\mathrm{CO}$ line data from the archives of JCMT. It is found that $\mathrm{CO}$ lines display red and blueshifted velocity profiles which can be attributed to the emission arising from distinct components of the $\mathrm{CO}$ gas moving in the opposite direction from the central core. In order to get a picture of the spatial extent of the outflow in the vicinity of G12.42+0.50, we construct the zeroth-moment map of the two components, which is shown in Fig. 7. The image reveals two distinct, spatially separated red and blue lobes. The radio emission and the SMA cores are located towards the centroid of the detected bipolar outflow.

\section{Summary}

The comprehensive multiwavelength study of G12.42+0.50 presented here shows this complex to be an active high-mass star-forming region in an early evolutionary phase confirmed by conclusive signatures of infall and outflow activities. The ionized emission probed with GMRT suggests the coexistence of an UC H II region and an ionized thermal jet driven by the same MYSO.

\section{References}



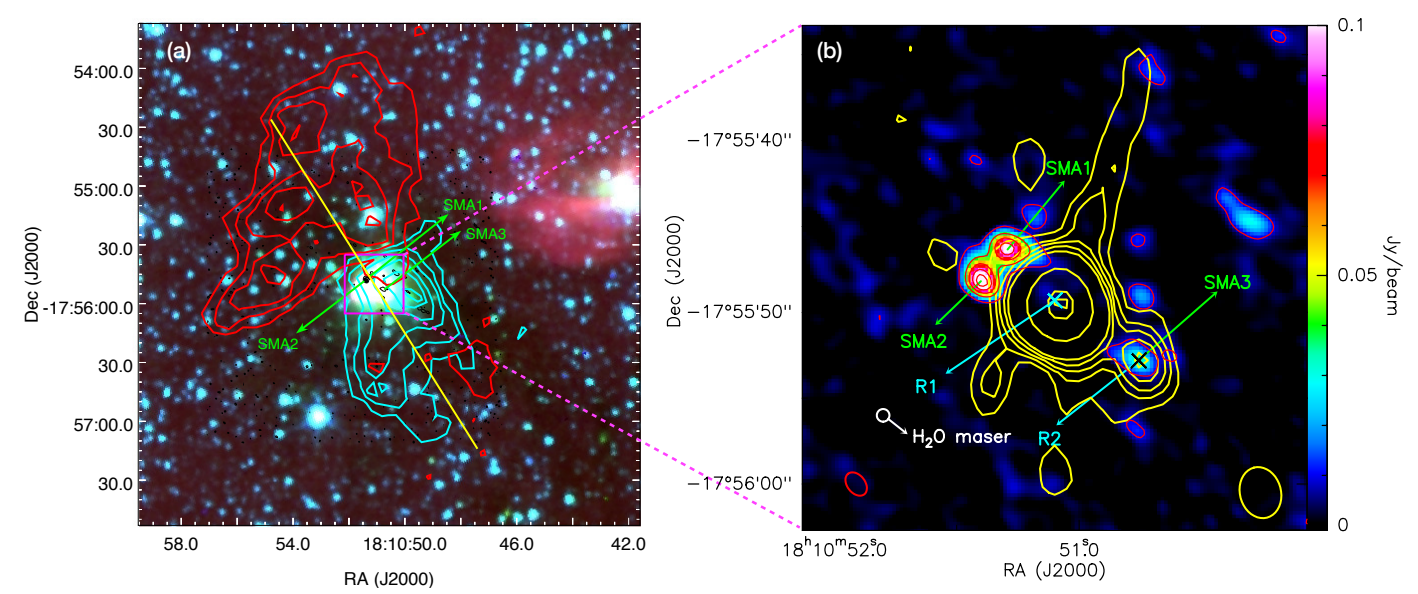

Figure 7: (a) The Spitzer IRAC colour composite image of G12.42+0.50, overlaid with the SMA $1.1 \mathrm{~mm}$ emission contours in black with the contour levels 3,12, 21, 30 and 39 times $\sigma(\sigma \sim$ $3 \mathrm{mJy} / \mathrm{beam})$. The CO $(3-2)$ emission integrated from the peak of the blueshifted profile to the blue wings is represented using cyan contours and from the peak of the red profile to the red wings is represented using red contours. The contours start from the $5 \sigma$ level for both the red and blue lobes and increases in steps of $3 \sigma$ and $4 \sigma$, respectively $\left(\sigma=2.7 \mathrm{~K} \mathrm{~km} \mathrm{~s}^{-1}\right.$ for red lobe and $\sigma=2.3 \mathrm{~K} \mathrm{~km} \mathrm{~s}^{-1}$ for blue lobe ). The red and blue lobes of the molecular outflow lie along a similar axis as the ionized jet. (b) The colour scale represents the $1.1 \mathrm{~mm}$ continuum emission from SMA observed towards $\mathrm{G} 12.42+0.50$. Radio emission at $1390 \mathrm{MHz}$ is represented by yellow contours with the contour levels same as that in Fig. 1(a). The restoring beams of the $1390 \mathrm{MHz}$ map and $1.1 \mathrm{~mm}$ map are indicated at the bottom- right and left of the image, respectively. The ' $\mathrm{x}$ 's indicate the positions of R1 and R2. The white circle marks the position of the $\mathrm{H}_{2} \mathrm{O}$ maser in the vicinity of $\mathrm{G} 12.42+0.50$. This figure is taken from Issac et al. (2019).

Anglada G., 1996, in Astronomical Society of the Pacific Conference Series, Vol. 93, Radio Emission from the Stars and the Sun, Taylor A. R., Paredes J. M., eds., pp. 314

Battersby C., Bally J., Ginsburg A. et al. 2011, A\&A, 535, A128

Beltrán M. T., Cesaroni R., Moscadelli L. et al. 2016, A\&A, 593, A49

Benjamin R. A., Churchwell E., Babler B. L. et al. 2003, PASP, 115, 953

Caratti o Garatti A., Stecklum B., Linz H. et al. 2015, A\&A, 573, A82

Chambers E. T., Jackson J. M., Rathborne J. M. et al. 2009, ApJS, 181, 360

Chen X., Shen Z.-Q., Li J.-J. et al. 2010, ApJ, 710, 150

Cyganowski C. J., Whitney B. A., Holden E. et al. 2008, AJ, 136, 2391

Foster J. B., Jackson J. M., Barnes P. J. et al. 2011, ApJS, 197, 25

Guzmán A. E., Garay G., Rodrguez L. F. et al. 2016, ApJ, 826, 208

Issac N., Tej A., Liu T. et al. 2019, MNRAS, 485, 1775

Jackson J. M., Rathborne J. M., Foster J. B. et al. 2013, PASA, 30, 57

Jaffe D. T., Hildebrand R. H., Keene J. et al. 1984, ApJ, 281, 225

Kauffmann J., Bertoldi F., Bourke T. L. et al. 2008, A\&A, 487, 993

Lee H.-T., Takami M., Duan H.-Y. et al. 2012, ApJS, 200, 2

Lee H.-T., Liao W.-T., Froebrich D. et al. 2013, ApJS, 208, 23

Rodriguez L. F., 1997, in IAU Symposium, Vol. 182, Herbig-Haro Flows and the Birth of Stars, Reipurth B., Bertout C., eds., pp. 8392

Urquhart J. S., Hoare M. G., Purcell C. R. et al. 2009, A\&A, 501, 539

Ward-Thompson D., Robson E. I. 1990, MNRAS, 244, 458

Wu Y., Henkel C., Xue R. et al. 2007, ApJ, 669, L37 